\title{
AN INTERMEDIATE ASYMPTOTIC SOLUTION OF THE COUPLED CREEP-DAMAGE CRACK PROBLEM IN SIMILARITY VARIABLES
}

\author{
Larisa V. Stepanova \\ Samara State University, 443011, Akad. Pavlov str., 1, Samara, Russia
}

\begin{abstract}
The class of self-similar solutions to coupled (creep-damage) crack problems is considered. The constitutive model is based on continuum damage mechanics. The conventional Kachanov-Rabotnov creep-damage theory is utilized to study the asymptotic behavior of damage in the region very near the crack tip. The totally damaged zone where the damage (integrity) parameter reaches its critical value is assumed to exist in the vicinity of the crack tip. Using the similarity variable the asymptotic solutions to mode I and mode III crack problems are obtained. The asymptotic stress, creep strain rate and damage fields near the crack tip are analyzed by solving nonlinear eigenvalue problems resulting in a new far stress distribution. The configurations of the totally damaged zone governed by the new far stress field are found and analyzed.
\end{abstract}

\section{INTRODUCTION}

Analysis of effects of material damage on the stress and strain fields near crack tip in non-linear materials is the very important problem for evaluation of crack behavior in elements of structures. The influence of damage on crack-tip fields has been the subject of many papers, especially for cracks in brittle materials [1], elastic-plastic-brittle cracks $[2,3]$, creep cracks [4-6] and fatigue cracks [7]. Thus, the phenomenon of crack growth in materials undergoing deformations coupled with damage has been investigated extensively over the past twenty years. Some of the essential aspects of the considered set of twodimensional crack problems and the results obtained can be highlighted. 1 . The damage gives significant influence on the stress and strain (strain rate) fields near the crack tip. 2. The mathematical structure of governing equations is affected by the modelling of damage. 3. While the Hutchinson-Rice-Rosengren (HRR) - field of non-linear fracture mechanics always shows the stress singularity at the crack tip for any finite value of the stress exponent, the preceding material damage in front of the crack tip decreases the singularity, and may give non-singular stress field. 4. The totally damage and (or) active damage zone (process zone) need be modelled in the crack tip region.

In the present work the asymptotic stress, strain rate and continuity fields in the vicinity of mode I and mode III cracks in damaged materials are obtained using the self-similar variable proposed by Riedel [8]. The form of the similarity solution has been introduced by Riedel. However there exist no solutions where the similarity property of damage mechanics equations is used. The advantage of a similarity solution is that it 
reduces the number of independent variables in the problem by one. This simplification allows us to gain insight into the time evolution of the near tip stress fields and the far field boundary condition.

In discussing crack growth on the basis of damage mechanics it is advantageous to introduce the term "totally damaged zone". The totally damaged zone can be interpreted as a zone occupied by microcracks oriented orthogonally to the main crack. Inside the totally damaged zone (TDZ) the damage involved reaches its critical value (for instance, the damage parameter reaches unity) and a complete fracture failure occurs. In view of material damage stresses are relaxed to vanishing. Therefore one can assume that the stress tensor components in the TDZ equal zero. Outside the zone damage alters the stress distribution substantially compared to the corresponding non-damaging material. Well outside that zone the damage parameter is equal to 1.

In the present study mode I and III crack problems for power-law creeping materials are considered by employing the self-similar variable on the assumption that the TDZ in the vicinity of the crack tip exists.

\section{SIMILARITY SOLUTIONS TO DAMAGE-MECHANICS EQUATIONS}

Let us consider a semi-infinite crack in a material with constitutive equations formulated in the framework of continuum damage mechanics. The constitutive model to be employed is the phenomenological model of Kachanov and Rabotnov. The integrity (continuity) parameter $\psi$ is assumed to affect the stress - strain rate relation according to

$$
\dot{\varepsilon}_{i j}=(3 / 2) B\left(\sigma_{e} / \psi\right)^{n-1} s_{i j} / \psi,
$$

where $\dot{\varepsilon}_{i j}$ is the creep strain rates, $s_{i j}=\sigma_{i j}-\sigma_{k k} \delta_{i j} / 3$ is the deviatoric stresses, $\sigma_{e}=$ $\sqrt{3 s_{i j} s_{i j} / 2}$ is the equivalent tensile stress, $B$ is the creep coefficient and $n$ is the creep exponent of Norton's creep law. According to the conventional creep-damage theory of Kachanov-Rabotnov the integrity parameter $\psi$ evolutes as follows

$$
d \psi / d t=-A\left(\sigma_{e q v} / \psi\right)^{m}, \quad \sigma_{e q v}=\alpha \sigma_{1}+\beta \sigma_{e}+(1-\alpha-\beta) \sigma,
$$

where $A, m(m>0)$ are material parameters, $\sigma_{e q v}$ is the damage equivalent stress, $\sigma_{1}$ is the maximum principal stress, $\sigma=\sigma_{k k}$ is the hydrostatic stress. Asymptotic remote boundary conditions have the form

$$
\sigma_{i j}(r \rightarrow \infty, \theta, t)=\left[C^{*} /\left(B I_{n} r\right)\right]^{1 /(n+1)} \bar{\sigma}_{i j}(\theta, n),
$$

where $r$ and $\theta$ are polar coordinates centered at the crack tip. The dimensionless constant $I_{n}$ and the $\theta$-variation functions of the suitably normalized functions $\bar{\sigma}_{i j}$ depend only on the creep exponent $n$.

If damage develops in the region which is small compared to the creep zone (the small scale damage conception), the boundary conditions (3) require that the stress field must approach the Hutchinson-Rice-Rosengren (HRR) field at large distances from the crack tip.

Dimensional analysis of Eqs. (1) - (3) shows that the damage mechanics equations must have similarity solutions of the form [8]

$$
\sigma_{i j}(r, \theta, t)=(A t)^{-1 / m} \Sigma_{i j}(R, \theta), \quad \psi(r, \theta, t)=\psi(R, \theta)
$$


with the similarity variable

$$
R=r(A t)^{-(n+1) / m} B I_{n} / C^{*} .
$$

The dimensionless functions $\Sigma_{i j}(R, \theta)$ and $\psi(R, \theta)$ are as yet unknown. The validity of these similarity presentations of the solutions should be verified by insertion of (4) into governing equations and boundary conditions.

It should be noted that the asymptotic remote boundary condition can be formulated in a more general form

$$
\sigma_{i j}(r, \theta, t) \rightarrow \tilde{C} r^{s} \bar{\sigma}_{i j}(\theta, n) .
$$

Thus, if the far stress field is determined by the HRR-solution then $\tilde{C}=\left(C^{*} /\left(B I_{n}\right)\right)^{1 /(n+1)}$ and $s=-1 /(n+1)$. If damage develops within the region of dominance of the $K$ field (where $K$ is the stress intensity factor), the remote boundary condition is

$$
\sigma_{i j}(r, \theta, t) \rightarrow(K / \sqrt{2 \pi r}) \bar{\sigma}_{i j}(\theta),
$$

where $\bar{\sigma}_{i j}(\theta)$ are normalized universal functions describing the angular variation of the stress components and $s=-1 / 2, \tilde{C}=K / \sqrt{2 \pi r}$.

Dimensional analysis of (1), (2) and (6) shows that the damage mechanics equations must have similarity solutions of the same form (4), where the similarity variable is expressed as

$$
R=r\left(A t \tilde{C}^{m}\right)^{1 /(s m)}
$$

where the exponent shas to be found.

\section{ANTIPLANE SHEAR CRACK. BASIC EQUATIONS AND ASYMPTOTIC SOLUTION}

A static mode III crack problem in a damaged creeping medium is considered. The equilibrium and compatibility equations in the polar coordinate system in terms of the similarity variable (8) can, respectively, be written as

$$
\frac{\partial}{\partial R}\left(R \Sigma_{R z}\right)+\frac{\partial \Sigma_{\theta z}}{\partial \theta}=0, \quad \frac{\partial}{\partial R}\left(R \Gamma_{\theta z}\right)=\frac{\partial \Gamma_{R z}}{\partial \theta},
$$

where $\Gamma_{i j}(R, \theta)=2 \dot{\varepsilon}_{i j}(r, \theta, t)(A t)^{-n /(s m)} /(3 B)$. The kinetic equation (2) takes the form

$$
R \partial \psi / \partial R=-s m(\Sigma / \psi)^{m}, \quad \Sigma^{2}=\Sigma_{R z}^{2}+\Sigma_{\theta z}^{2} .
$$

The traction-free condition on the crack surface yields

$$
\Sigma_{\theta z}(R, \theta=\pi)=0 .
$$

By virtue of symmetry the vanishing condition at $\theta=0$ holds

$$
\Sigma_{R z}(R, \theta=0)=0 .
$$
variable as

The remote boundary condition (6) can be expressed in terms of the similarity

$$
\Sigma_{i j}(R, \theta) \rightarrow R^{s} \bar{\sigma}_{i j}(\theta, n)
$$


It is assumed that the totally damaged zone where the integrity parameter reaches its critical value and all the stress tensor components are equal to zero appears in the vicinity of the crack tip. To obtain the shape of the totally damaged zone one can find the solution at large distances from the crack tip (at large distances compared with the crack length, the specimen sizes but at yet small distances compared with the characteristic length of the totally damaged zone).

The asymptotic expansions of the effective stress (the stress referred to the surface that really transmits the internal forces) and the integrity parameter for large distances $(R \rightarrow \infty)$ from the crack tip are supposed to be separable and can be expressed as series as

$$
\begin{aligned}
& \frac{\Sigma_{i j}}{\psi}(R, \theta)=R^{s} f_{i j}^{(0)}+R^{s_{1}} f_{i j}^{(1)}+R^{s_{2}} f_{i j}^{(2)}+R^{s_{3}} f_{i j}^{(3)}+R^{s_{4}} f_{i j}^{(4)}+o\left(R^{s_{4}}\right), \\
& \psi(R, \theta)=1-R^{\gamma_{1}} g^{(1)}-R^{\gamma_{2}} g^{(2)}-R^{\gamma_{3}} g^{(3)}-R^{\gamma_{4}} g^{(4)}-R^{\gamma_{5}} g^{(5)}+o\left(R^{\gamma_{5}}\right)
\end{aligned}
$$

where $f_{i j}^{(k)}=f_{i j}^{(k)}(\theta), g^{(k)}=g^{(k)}(\theta)$; the index $0,1,2,3,4,5 \ldots$ correspond to the zeroorder, first-order, second-order, third-order, forth-order and fifth-order fields, respectively, $s>s_{1}>s_{2}>s_{3}>s_{4}>\ldots, \gamma_{1}>\gamma_{2}>\gamma_{3}>\gamma_{4}>\gamma_{5}>\ldots$. Based on the asymptotic analysis of the kinetic equation (10) one has $\gamma_{1}=s m, \gamma_{k}=s_{k-1}-s+s m, k>1$.

The asymptotic expansions of stress components are directly obtained from Eqs.

$$
\begin{aligned}
\Sigma_{i j}(R, \theta)= & R^{s} \tau_{i j}^{(0)}(\theta)+R^{s+s m} \tau_{i j}^{(1)}(\theta)+R^{s+2 s m} \tau_{i j}^{(2)}(\theta)+R^{s+3 s m} \tau_{i j}^{(3)}(\theta) \\
& +R^{s+4 s m} \tau_{i j}^{(4)}(\theta)+o\left(R^{s+4 s m}\right) \\
s_{k}= & s+k s m .
\end{aligned}
$$

where $\tau_{i j}^{(0)}(\theta)=f_{i j}^{(0)}(\theta), \quad \tau_{i j}^{(k)}(\theta)=f_{i j}^{(k)}(\theta)-\sum_{l=1}^{k} f_{i j}^{(k-l)}(\theta) g^{(l)}(\theta), \quad k \geq 1$. The multi-term asymptotic expansion of the effective stress has the form

$$
\Sigma / \psi=R^{s} f\left(1+R^{s m} \tau^{(1)}+R^{2 s m} \tau^{(2)}+R^{3 s m} \tau^{(3)}+R^{4 s m} \tau^{(4)}\right)+o\left(R^{s+4 s m}\right),
$$

where

$$
\begin{aligned}
& \tau^{(1)}=\tau^{(1)}(\theta)=f_{1} f^{-2} / 2, f^{2}=\left(f_{R z}^{(0)}\right)^{2}+\left(f_{\theta z}^{(0)}\right)^{2}, \quad f_{k}(\theta)=\sum_{l=0}^{k} f_{i z}^{(l)} f_{i z}^{(k-l)}, \quad i=R, \theta, \\
& \tau^{(2)}=\left(f_{2}-(1 / 4) f_{1}^{2} f^{-2}\right) f^{-2} / 2, \tau^{(3)}=\left[f_{3}-(1 / 2) f_{1} f_{2} f^{-2}+(1 / 8) f_{1}^{3} f^{-4}\right] f^{-2} / 2, \\
& \tau^{(4)}=\left[f_{4}-(1 / 2) f_{1} f_{3} f^{-2}-(1 / 4) f_{2}^{2} f^{-2}+(3 / 8) f_{1}^{2} f_{2} f^{-4}-(5 / 64) f_{1}^{3} f^{-4}\right] f^{-2} / 2 .
\end{aligned}
$$

Substituting the stress expansion (15) into the equilibrium equation (9) one obtains the sequence of ordinary differential equations with respect to $f_{i j}^{(k)}(\theta)$

$$
\tau_{\theta z, \theta}^{(k)}+(s+k s m+1) \tau_{R z}^{(k)}=0 .
$$


The fifth-term asymptotic expansion of the creep strain rate components is given as

$$
\begin{aligned}
& \Gamma_{i z}(R, \theta)=R^{s n} \gamma_{i z}^{(0)}(\theta)+R^{s(n+m)} \gamma_{i z}^{(1)}(\theta)+R^{s(n+2 m)} \gamma_{i z}^{(2)}(\theta)+ \\
& +R^{s(n+3 m)} \gamma_{i z}^{(3)}(\theta)+R^{s(n+4 m)} \gamma_{i z}^{(4)}(\theta)+o\left(R^{s(n+4 m)}\right),
\end{aligned}
$$

where $\gamma_{i z}^{(0)}(\theta)=f^{n-1} f_{i z}^{(0)}(\theta), \quad \gamma_{i z}^{(k)}(\theta)=f^{n-1}\left[f_{i z}^{(k)}(\theta)+\sum_{l=1}^{k} c_{l}(\theta) f_{i z}^{(k-l-1)}(\theta)\right]$,

$$
\begin{aligned}
c_{1}= & (n-1) \tau^{(1)}, \quad c_{2}=(n-1)\left[\tau^{(2)}+(1 / 2)(n-2)\left(\tau^{(1)}\right)^{2}\right], \\
c_{3}= & (n-1)\left[\tau^{(3)}+(n-2) \tau^{(1)} \tau^{(2)}+(1 / 6)(n-2)(n-3)\left(\tau^{(1)}\right)^{3}\right], \\
c_{4}= & (n-1)\left[\tau^{(4)}+(n-2) \tau^{(1)} \tau^{(3)}+(1 / 2)(n-2)\left(\tau^{(2)}\right)^{2}\right. \\
& \left.+(1 / 2)(n-2)(n-3)\left(\tau^{(1)}\right)^{2} \tau^{(2)}+(1 / 24)(n-2)(n-3)(n-4)\left(\tau^{(1)}\right)^{4}\right] .
\end{aligned}
$$

By inserting the asymptotic expansions (18) into the compatibility equation (9) one obtains the sequence of the ordinary differential equations

$$
\gamma_{R z, \theta}^{(k)}=[s(n+k m)+1] \gamma_{\theta z}^{(k)} .
$$

The damage evolution equation (10) allows us to find the relations among the angular integrity parameter functions and the angular effective stress functions

$$
\begin{aligned}
g^{(1)}(\theta)= & f^{m}, g^{(2)}(\theta)=\frac{1}{2} m f^{m} \tau^{(1)}, \\
g^{(3)}(\theta)= & \frac{1}{3} m f^{m}\left[\tau^{(2)}+\frac{1}{2}(m-1)\left(\tau^{(1)}\right)^{2}\right], \\
g^{(4)}(\theta)= & \frac{1}{4} m f^{m}\left[\tau^{(3)}+(m-1) \tau^{(1)} \tau^{(2)}+\frac{1}{6}(m-1)(m-2)\left(\tau^{(1)}\right)^{3}\right], \\
g^{(5)}(\theta)= & \frac{1}{5} m f^{m}\left[\tau^{(4)}+(m-1) \tau^{(1)} \tau^{(3)}+\frac{1}{2}(m-1)\left(\tau^{(2)}\right)^{2}\right. \\
& \left.+\frac{1}{2}(m-1)(m-2)\left(\tau^{(1)}\right)^{2} \tau^{(2)}+\frac{1}{24}(m-1)(m-2)(m-3)\left(\tau^{(1)}\right)^{4}\right] .
\end{aligned}
$$

Eqs. (17), (19) and (20), where $k=0,1,2,3,4$, are the final governing equations for the zero-order, first-order, second-order, third-order and fourth-order asymptotic stress fields. The system of Eqs. (17), (19) with the boundary conditions following from (11) and (12) has been numerically integrated by a Runge-Kutta-Fehlberg method. After integration it is possible to determine the shape of the TDZ by means of the asymptotic equation

$$
\psi(R, \theta)=1-R^{s m} g^{(1)}-R^{2 s m} g^{(2)}-R^{3 s m} g^{(3)}-R^{4 s m} g^{(4)}-R^{5 s m} g^{(5)}=0
$$

because it is assumed that on the boundary of the TDZ the integrity parameter reaches its critical value. It is interesting to compare the boundaries of the TDZ given by the two-term, three-term, four-term, five-term and six-term asymptotic integrity parameter expansion. It is turned out that if the remote boundary condition is postulated as the condition of 
the asymptotic approaching the HRR-fields (the remote boundary condition (3)) then the shapes of the TDZ given by the two-term and three-term asymptotic expansions differ essentially from each other. Figure 1 shows the shapes of the TDZ determined by the two-term and three-term asymptotic expansions in this case. Moreover it is not possible to evaluate the boundary of the TDZ given by the four-term asymptotic integrity parameter expansion since there are no roots of the algebraic equation

$$
\psi(R, \theta)=1-R^{s m} g^{(1)}(\theta)-R^{2 s m} g^{(2)}(\theta)-R^{3 s m} g^{(3)}(\theta)=0
$$

corresponding to the physical meaning of the similarity variable (5).
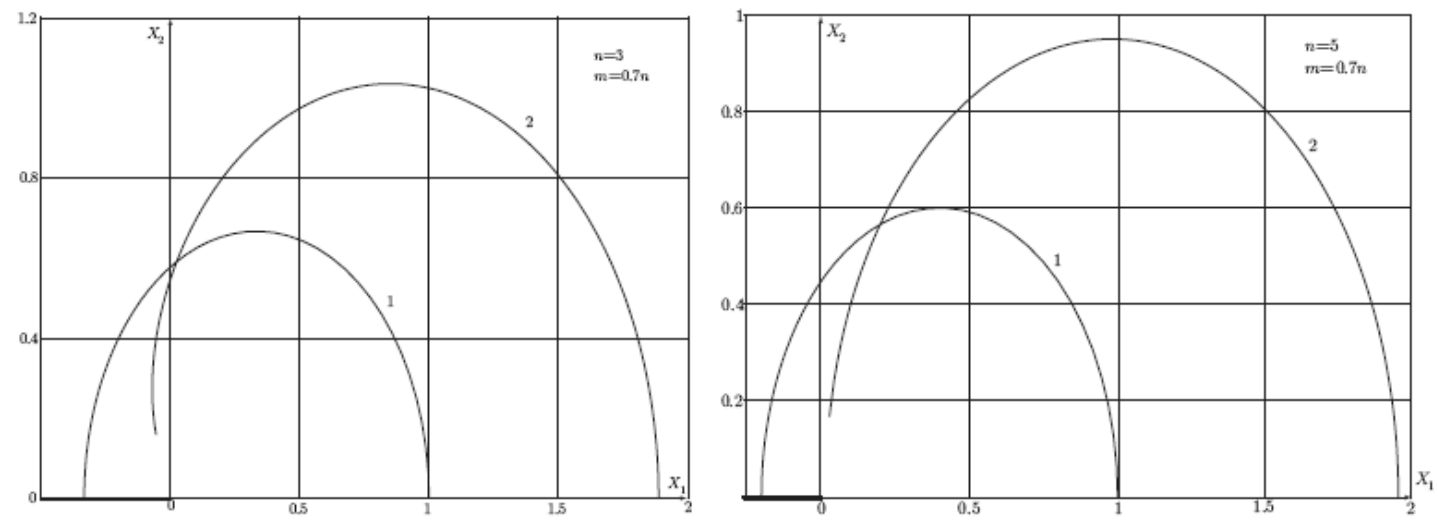

Fig. 1. The contours of the TDZ in the vicinity of the antiplane shear crack tip: 1 - the contour given by the two-term asymptotic integrity parameter expansion, 2 - the contour given by the three-term asymptotic integrity parameter expansion

To elucidate the cause of this fact one can formulate the remote boundary condition in a more general form (13) with an unknown exponent $s$ which must be found as a part of the solution under the condition of convergence of the TDZ boundary to some limit contour.

It should be noted that Eqs. (17) and (19) for $k=0$ in conjunction with the boundary conditions describe a nonlinear eigenvalue problem

$$
\begin{aligned}
& \frac{d f_{R z}^{(0)}}{d \theta}=f_{\theta z}^{(0)} \frac{(s n+1) f^{2}+(n-1)(s+1)\left(f_{R z}^{(0)}\right)^{2}}{(n-1)\left(f_{R z}^{(0)}\right)^{2}+f^{2}}, \\
& \frac{d f_{\theta z}^{(0)}}{d \theta}=-(s+1) f_{R z}^{(0)}, \quad f_{\theta z}^{(0)}(\theta=\pi)=0, \quad f_{R z}^{(0)}(\theta=0)=0 .
\end{aligned}
$$

where the unknown eigenvalue $s$ and the eigenfunctions $f_{i z}^{(0)}(\theta)$ depend on the boundary conditions and the creep exponent. One eigenvalue corresponding to the HRR-problem $s=-1 /(n+1)$ is well-known. Previously $[9,10]$ the whole eigenspectrum of the nonlinear 
eigenvalue problem considered was established

$\lambda=\frac{n\left(\lambda_{0}^{2}+2 \lambda_{0}-1\right)+\left(\lambda_{0}-1\right)^{2}}{2 n\left(2 \lambda_{0}-1\right)}+\frac{\sqrt{\left[n\left(\lambda_{0}^{2}+2 \lambda_{0}-1\right)+\left(\lambda_{0}-1\right)^{2}\right]^{2}-4 n^{2} \lambda_{0}^{2}\left(2 \lambda_{0}-1\right)}}{2 n\left(2 \lambda_{0}-1\right)}$,

where $\lambda=s+1, \lambda_{0}$ refers to the linear problem. For the HRR-problem the eigenvalue corresponding to the linear problem is $\lambda_{0}=1 / 2$.

Thus, it is necessary to find an eigenvalue different from the eigenvalue of the HRRproblem and resulting in the boundaries of the TDZ which are convergent to some limit contour. The eigenvalues required are listed in Table 1.

Table 1. The eigenvalue $s$ for different values of material constants

\begin{tabular}{|c|c|}
\hline$n=m=1$ & $s=-1.5$ \\
\hline$n=2, m=0.7 n$ & $s=-1.230291$ \\
\hline$n=3, m=0.7 n$ & $s=-1.183013$ \\
\hline$n=4, m=0.7 n$ & $s=-1.164790$ \\
\hline$n=5, m=0.7 n$ & $s=-1.155234$ \\
\hline$n=6, m=0.7 n$ & $s=-1.149367$ \\
\hline$n=7, m=0.7 n$ & $s=-1.145402$ \\
\hline$n=8, m=0.7 n$ & $s=-1.142544$ \\
\hline$n=9, m=0.7 n$ & $s=-1.140388$ \\
\hline$n=10, m=0.7 n$ & $s=-1.138703$ \\
\hline
\end{tabular}

The configurations of the TDZ for the new far field stress asymptotics are shown in Figures 2, 3, where the following notations are accepted: 1 - the contour given by the two-term asymptotic expansion of the integrity parameter, 2 - the contour given by the three-term asymptotic expansion of the integrity parameter, 3 - the contour given by the four-term asymptotic expansion of the integrity parameter, 4 - the contour given by the five-term asymptotic expansion of the integrity parameter, 5 - the contour given by the six-term asymptotic expansion of the integrity parameter. It is clearly seen from Figure 2 and 3 that the boundaries of the TDZ converge to the limit contour.

\section{FINITE DIFFERENCE SOLUTION OF MODE III CRACK PROBLEM}

To justify the asymptotic solution obtained one can address to the direct numerical integration of Eqs. (9) - (13) formulated in terms of the similarity variable. The numerical solution has been found by the finite difference method. The results of the numerical calculations are presented by Figures 4 and 5 . The stress and integrity distributions are shown in Figure 4. It is interesting to represent the effective stress in double logarithmic coordinates (Figure 5). It is seen that there are two rectilinear parts: one linear region corresponds to the HRR-filed while the order linear part corresponds to the new intermediate asymptotic solution. The intermediate asymptotic solution is the stress and integrity distributions valid for times and distances at which the influence of fine details of initial and boundary conditions is lost [11-14]. 

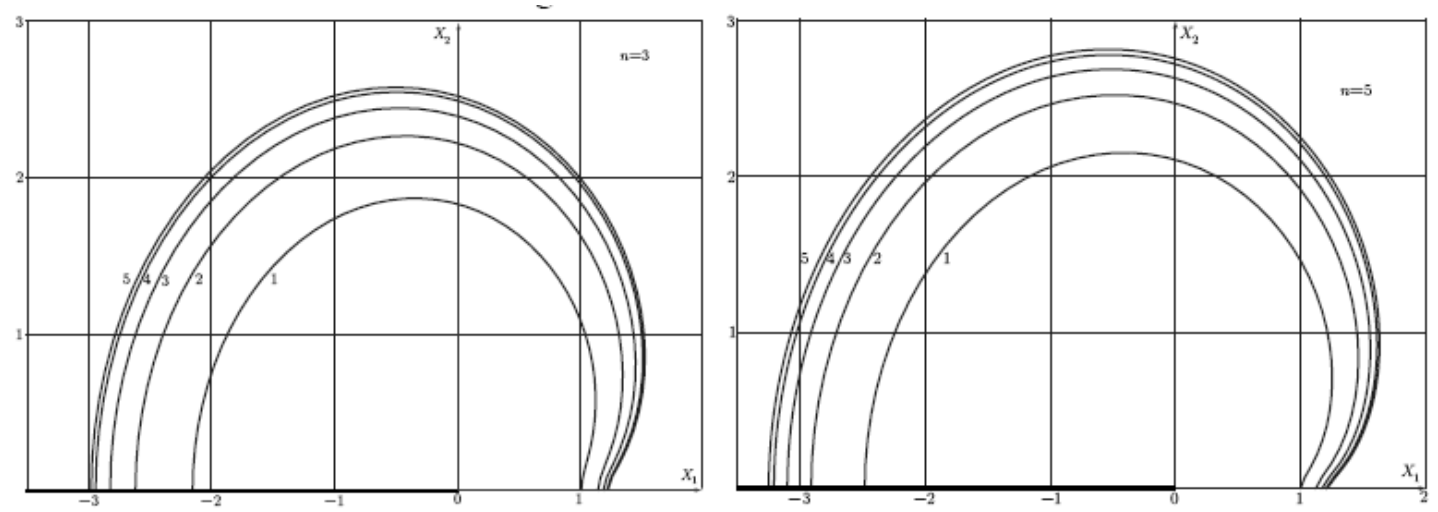

Fig. 2. The contours of the TDZ for $n=3, m=0.7 n$ and $n=5, m=0.7 n$
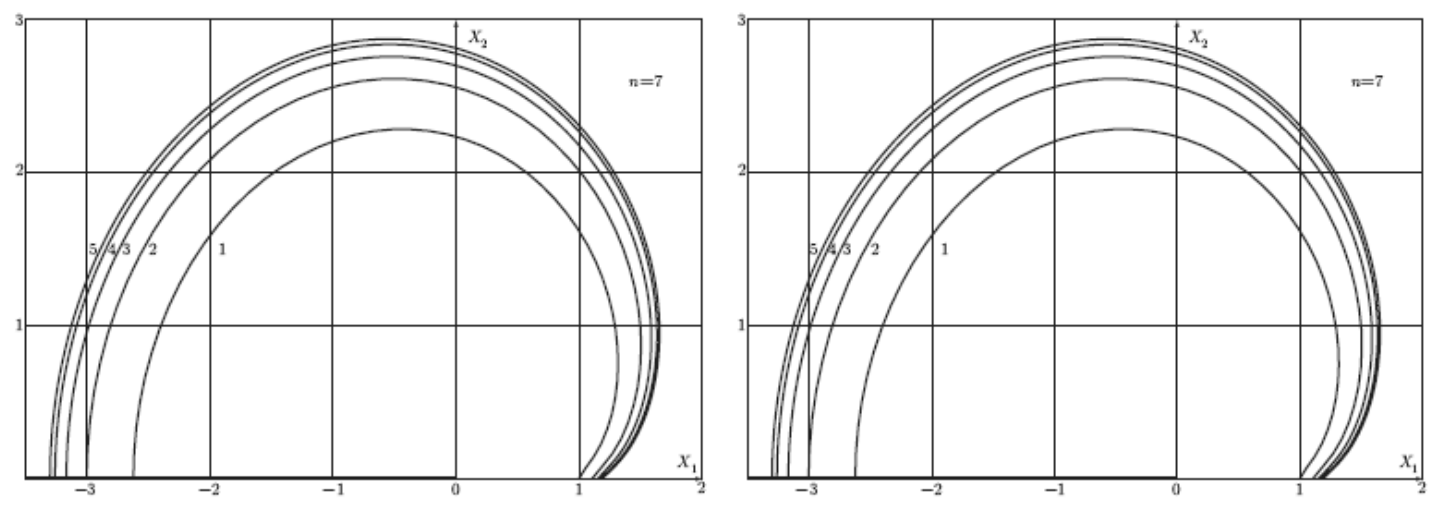

Fig. 3. The contours of the TDZ for $n=7, m=0.7 n$ and $n=9, m=0.7 n$
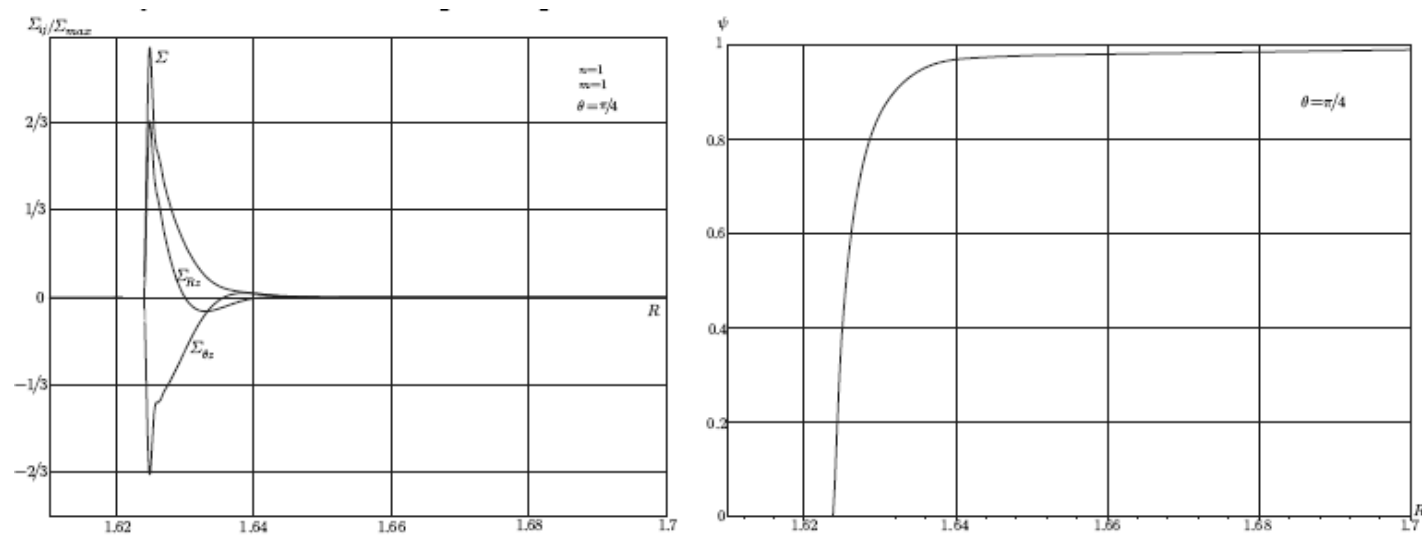

Fig. 4. Stresses and integrity distributions in the vicinity of the antiplane shear crack 

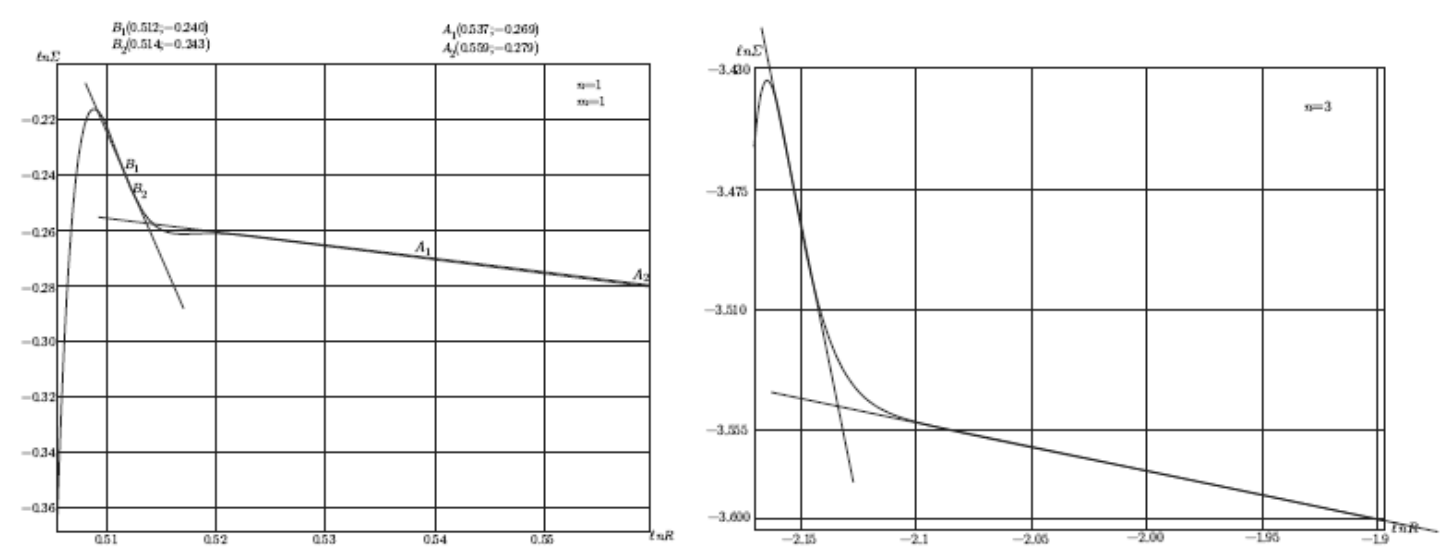

Fig. 5. Logarithmic plot of the effective stress showing that $\Sigma$ is proportional to the similarity variable $R$

\section{MODE I CRACK. FUNDAMENTAL EQUATIONS AND ASYMPTOTIC SOLUTION}

A static mode I crack problem in a damaged creeping material under the plane strain and plane stress conditions is considered. The equilibrium and compatibility equations in the polar coordinate system can, respectively, be written as

$$
\begin{gathered}
\frac{\partial \sigma_{r r}}{\partial r}+\frac{1}{r} \frac{\partial \sigma_{r \theta}}{\partial \theta}+\frac{\sigma_{r r}-\sigma_{\theta \theta}}{r}=0, \frac{\partial \sigma_{r \theta}}{\partial r}+\frac{1}{r} \frac{\partial \sigma_{\theta \theta}}{\partial \theta}+2 \frac{\sigma_{r \theta}}{r}=0 \\
2 \frac{\partial}{\partial r}\left(r \frac{\partial \dot{\varepsilon}_{r \theta}}{\partial \theta}\right)=\frac{\partial^{2} \dot{\varepsilon}_{r r}}{\partial \theta^{2}}-r \frac{\partial \dot{\varepsilon}_{r r}}{\partial r}+r \frac{\partial^{2}\left(r \dot{\varepsilon}_{\theta \theta}\right)}{\partial r^{2}}
\end{gathered}
$$

The creep power-law constitutive equations in the coupled creep-damage formulation are described by

$$
\dot{\varepsilon}_{r r}=-\dot{\varepsilon}_{\theta \theta}=\frac{3}{4} B\left(\frac{\sigma_{e}}{\psi}\right)^{n-1} \frac{\sigma_{r r}-\sigma_{\theta \theta}}{\psi}, \quad \dot{\varepsilon}_{r \theta}=\frac{3}{2} B\left(\frac{\sigma_{e}}{\psi}\right)^{n-1} \frac{\sigma_{r \theta}}{\psi},
$$

where $\sigma_{e}^{2}=3\left(\sigma_{r r}-\sigma_{\theta \theta}\right)^{2} / 4+3 \sigma_{r \theta}^{2}$ for plane strain conditions,

$$
\begin{aligned}
& \dot{\varepsilon}_{r r}=\frac{B}{2}\left(\frac{\sigma_{e}}{\psi}\right)^{n-1} \frac{2 \sigma_{r r}-\sigma_{\theta \theta}}{\psi}, \\
& \dot{\varepsilon}_{\theta \theta}=\frac{B}{2}\left(\frac{\sigma_{e}}{\psi}\right)^{n-1} \frac{2 \sigma_{\theta \theta}-\sigma_{r r}}{\psi}, \\
& \dot{\varepsilon}_{r \theta}=\frac{3 B}{2}\left(\frac{\sigma_{e}}{\psi}\right)^{n-1} \frac{\sigma_{r \theta}}{\psi}
\end{aligned}
$$

where $\sigma_{e}^{2}=\sigma_{r r}^{2}+\sigma_{\theta \theta}^{2}-\sigma_{r r} \sigma_{\theta \theta}+3 \sigma_{r \theta}^{2}$ for plane stress conditions.

The traction-free conditions on the crack surfaces yield

$$
\sigma_{\theta \theta}(r, \theta= \pm \pi)=0, \quad \sigma_{r \theta}(r, \theta= \pm \pi)=0 .
$$


The remote boundary condition has the form (6).

Analogously to the antiplane shear crack problem one can introduce the similarity variable (8). The equilibrium and compatibility equations hold their forms while the kinetics evolution law of damage takes the following form

$$
R \partial \psi / \partial R=-\operatorname{sm}\left(\Sigma_{\text {eqv }} / \psi\right)^{m},
$$

where $\Sigma_{e q v}=\alpha \Sigma_{1}+\beta \Sigma_{e}+(1-\alpha-\beta) \Sigma$ is the damage equivalent stress in terms of the similarity variable.

The Airy stress potential $F(R, \theta)$ can be used to obtain

$$
\sigma_{\theta \theta}=F_{, r r}, \quad \sigma_{r r}=\Delta F-\sigma_{\theta \theta}, \quad \sigma_{r \theta}=-\left(r^{-1} F_{, \theta}\right)_{, r} .
$$

It is assumed that the Airy stress function and the integrity parameter at large distances from the crack tip $(R \rightarrow \infty)$ are separable and can be expressed as series as

$$
\begin{aligned}
& F(R, \theta)=R^{\lambda+1} f^{(0)}(\theta)+R^{\lambda_{1}+1} f^{(1)}(\theta)+R^{\lambda_{2}+1} f^{(2)}(\theta)+o\left(R^{\lambda_{2}+1}\right), \\
& \psi(R, \theta)=1-R^{\gamma_{1}} g^{(1)}(\theta)-R^{\gamma_{2}} g^{(2)}(\theta)-R^{\gamma_{3}} g^{(3)}(\theta)+o\left(R^{\gamma_{3}}\right),
\end{aligned}
$$

$\lambda>\lambda_{1}>\lambda_{2}>\ldots$. The multi-term asymptotic stress expansions can be written in the form

$$
\begin{aligned}
\sigma_{R R}(R, \theta) & =R^{s}\left[\lambda f^{(0)}+\left(f^{(0)}\right)^{\prime \prime}\right]+R^{s_{1}}\left[\lambda_{1} f^{(1)}+\left(f^{(1)}\right)^{\prime \prime}\right]+R^{s_{2}}\left[\lambda_{2} f^{(2)}+\left(f^{(2)}\right)^{\prime \prime}\right]+o\left(R^{s_{2}}\right), \\
\sigma_{\theta \theta}(R, \theta) & =R^{s} \lambda(\lambda-1) f^{(0)}+R^{s_{1}} \lambda_{1}\left(\lambda_{1}-1\right) f^{(1)}+R^{s_{2}} \lambda_{2}\left(\lambda_{2}-1\right) f^{(2)}+o\left(R^{s_{2}}\right), \\
\sigma_{r \theta}(R, \theta) & =R^{s}(1-\lambda)\left(f^{(0)}\right)^{\prime}+R^{s_{1}}\left(1-\lambda_{1}\right)\left(f^{(1)}\right)^{\prime}+R^{s_{2}}\left(1-\lambda_{2}\right)\left(f^{(2)}\right)^{\prime}+o\left(R^{s_{2}}\right),
\end{aligned}
$$

where $s=\lambda-1, s_{1}=\lambda_{1}-1, s_{2}=\lambda_{2}-1$.

The three-term asymptotic creep strain rate expansions for plane strain conditions are determined by the formulae

$$
\begin{aligned}
& \dot{\varepsilon}_{R R}(R, \theta)=-\dot{\varepsilon}_{\theta \theta}(R, \theta)=R^{s n} \varepsilon_{R R}^{(0)}(\theta)+R^{s(n+m)} \varepsilon_{R R}^{(1)}(\theta)+R^{s(n+2 m)} \varepsilon_{R R}^{(2)}(\theta)+\ldots, \\
& \dot{\varepsilon}_{R \theta}(R, \theta)=R^{s n} \varepsilon_{R \theta}^{(0)}(\theta)+R^{s(n+m)} \varepsilon_{R \theta}^{(1)}(\theta)+R^{s(n+2 m)} \varepsilon_{R \theta}^{(2)}(\theta)+\ldots,
\end{aligned}
$$

where

$$
\begin{aligned}
\varepsilon_{R R}^{(0)}(\theta)=f_{e}^{n-1}\left[\left(1-\lambda^{2}\right) f^{(0)}+\left(f^{(0)}\right)^{\prime \prime}\right], \varepsilon_{R \theta}^{(0)}(\theta)=-f_{e}^{n-1} \lambda\left(f^{(0)}\right)^{\prime}, \\
\varepsilon_{R R}^{(1)}=\frac{1}{2} f_{e}^{n-1}\left\{\left[\left(1-\lambda_{1}^{2}\right) f^{(1)}+\left(f^{(1)}\right)^{\prime \prime}\right]+\left[\left(1-\lambda^{2}\right) f^{(0)}+\left(f^{(0)}\right)^{\prime \prime}\right]\left[(n-1) f_{e}^{(1)}+n g^{(0)}\right]\right\}, \\
\varepsilon_{R \theta}^{(1)}=-f_{e}^{n-1}\left\{\lambda_{1}\left(f^{(1)}\right)^{\prime}+\lambda\left(f^{(0)}\right)^{\prime}\left[(n-1) f_{e}^{(1)}+n g^{(0)}\right]\right\}, \\
\varepsilon_{R R}^{(2)}=\frac{1}{2} f_{e}^{n-1}\left\{\left[\left(1-\lambda_{2}^{2}\right) f^{(2)}+\left(f^{(2)}\right)^{\prime \prime}\right]+\left[\left(1-\lambda_{1}^{2}\right) f^{(1)}+\left(f^{(1)}\right)^{\prime \prime}\right]\left[(n-1) f_{e}^{(1)}+n g^{(0)}\right]\right. \\
+\left[\left(1-\lambda^{2}\right) f^{(0)}+\left(f^{(0)}\right)^{\prime \prime}\right]\left[n\left(\frac{n+1}{2}\left(g^{(0)}\right)^{2}+g^{(1)}+(n-1) g^{(0)} f_{e}^{(1)}\right)\right. \\
\left.\left.+\frac{n-1}{2}\left((n-2)\left(f_{e}^{(1)}\right)^{2}+2 F_{e}^{2}\right)\right]\right\}
\end{aligned}
$$




$$
\begin{aligned}
& \varepsilon_{R \theta}^{(2)}=-f_{e}^{n-1}\left\{\lambda_{2}\left(f^{(2)}\right)^{\prime}+\lambda_{1}\left(f^{(1)}\right)^{\prime}\left[(n-1) f_{e}^{(1)}+n g^{(0)}\right]\right. \\
&+\lambda\left(f^{(0)}\right)^{\prime}\left[n\left(\frac{n+1}{2}\left(g^{(0)}\right)^{2}+g^{(1)}+(n-1) g^{(0)} f_{e}^{(1)}\right)\right. \\
&\left.\left.+\frac{n-1}{2}\left((n-2)\left(f_{e}^{(1)}\right)^{2}+2 F_{e}^{2}\right)\right]\right\}, \\
& f_{e}^{(1)}=\left\{\left[\left(1-\lambda^{2}\right) f^{(0)}+\left(f^{(0)}\right)^{\prime \prime}\right]\left[\left(1-\lambda_{1}^{2}\right) f^{(1)}+\left(f^{(1)}\right)^{\prime \prime}\right]+4 \lambda \lambda_{1}\left(f^{(0)}\right)^{\prime}\left(f^{(1)}\right)^{\prime}\right\} f_{e}^{-2}, \\
& F_{e}^{2}= \frac{1}{2}\left[f_{e}^{(2)}-\left(f_{e}^{(1)}\right)^{2}\right], \quad f_{e}^{(2)}=\left\{\left[\left(1-\lambda_{1}^{2}\right) f^{(1)}+\left(f^{(1)}\right)^{\prime \prime}\right]^{2}+4 \lambda_{1}^{2}\left[\left(f^{(1)}\right)^{\prime}\right]^{2}\right. \\
&\left.+2\left[\left(1-\lambda^{2}\right) f^{(0)}+\left(f^{(0)}\right)^{\prime \prime}\right]\left[\left(1-\lambda_{2}^{2}\right) f^{(2)}+\left(f^{(2)}\right)^{\prime \prime}\right]+8 \lambda \lambda_{2}\left(f^{(0)}\right)^{\prime}\left(f^{(2)}\right)^{\prime}\right\} f_{e}^{-2} .
\end{aligned}
$$

Using the asymptotic expansions (30) and the compatibility equation (23) one finds

$$
\begin{aligned}
& 2(s n+1) \varepsilon_{R \theta, \theta}^{(0)}=\varepsilon_{R R, \theta \theta}^{(0)}-s n(s n+2) \varepsilon_{R R}^{(0)}, \\
& 2[s(n+m)+1] \varepsilon_{R \theta, \theta}^{(1)}=\varepsilon_{R R, \theta \theta}^{(1)}-s(n+m)[s(n+m)+2] \varepsilon_{R R}^{(1)}, \\
& 2[s(n+2 m)+1] \varepsilon_{R \theta, \theta}^{(2)}=\varepsilon_{R R, \theta \theta}^{(2)}-s(n+m)[s(n+2 m)+2] \varepsilon_{R R}^{(2)} .
\end{aligned}
$$

Taking into account Eqs. (31) one can obtain the nonlinear ordinary differential equation with respect to $f^{(0)}(\theta)$ :

$$
\begin{aligned}
& f_{e}^{2}\left(f^{(0)}\right)^{I V}\left\{(n-1)\left[\left(1-\lambda^{2}\right) f^{(0)}+\left(f^{(0)}\right)^{\prime \prime}\right]^{2}+f_{e}^{2}\right\}+(n-1)(n-3) \\
& \times\left\{\left[\left(1-\lambda^{2}\right) f^{(0)}+\left(f^{(0)}\right)^{\prime \prime}\right]\left[\left(1-\lambda^{2}\right)\left(f^{(0)}\right)^{\prime}+\left(f^{(0)}\right)^{\prime \prime \prime}\right]+4 \lambda^{2}\left(f^{(0)}\right)^{\prime}\left(f^{(0)}\right)^{\prime \prime}\right\}^{2} \\
& \times\left[\left(1-\lambda^{2}\right) f^{(0)}+\left(f^{(0)}\right)^{\prime \prime}\right]+(n-1) f_{e}^{2}\left\{\left[\left(1-\lambda^{2}\right)\left(f^{(0)}\right)^{\prime}+\left(f^{(0)}\right)^{\prime \prime \prime}\right]^{2}\right. \\
& \left.+\left[\left(1-\lambda^{2}\right) f^{(0)}+\left(f^{(0)}\right)^{\prime \prime}\right]\left(1-\lambda^{2}\right)\left(f^{(0)}\right)^{\prime \prime}+4 \lambda^{2}\left[\left(f^{(0)}\right)^{\prime \prime 2}+\left(f^{(0)}\right)^{\prime}\left(f^{(0)}\right)^{\prime \prime \prime}\right]\right\} \\
& f_{e}^{2}\left(f^{(0)}\right)^{I V}\left\{(n-1)\left[\left(1-\lambda^{2}\right) f^{(0)}+\left(f^{(0)}\right)^{\prime \prime}\right]^{2}+f_{e}^{2}\right\}+(n-1)(n-3) \\
& \times\left[\left(1-\lambda^{2}\right) f^{(0)}+\left(f^{(0)}\right)^{\prime \prime}\right]+2(n-1) f_{e}^{2}\left\{\left[\left(1-\lambda^{2}\right) f^{(0)}+\left(f^{(0)}\right)^{\prime \prime}\right]\left[\left(1-\lambda^{2}\right)\left(f^{(0)}\right)^{\prime}+\left(f^{(0)}\right)^{\prime \prime \prime}\right]\right. \\
& \left.+4 \lambda^{2}\left(f^{(0)}\right)^{\prime}\left(f^{(0)}\right)^{\prime \prime}\right\}\left[\left(1-\lambda^{2}\right)\left(f^{(0)}\right)^{\prime}+\left(f^{(0)}\right)^{\prime \prime \prime}\right] \\
& +C_{1}(n-1) f_{e}^{2}\left\{\left[\left(1-\lambda^{2}\right) f^{(0)}+\left(f^{(0)}\right)^{\prime \prime}\right]\left[\left(1-\lambda^{2}\right)\left(f^{(0)}\right)^{\prime}+\left(f^{(0)}\right)^{\prime \prime \prime}\right]+4 \lambda^{2}\left(f^{(0)}\right)^{\prime}\left(f^{(0)}\right)^{\prime \prime}\right\} \\
& \times\left(f^{(0)}\right)^{\prime}+C_{1} f_{e}^{4}\left(f^{(0)}\right)^{\prime \prime}-C_{2} f_{e}^{4}\left[\left(1-\lambda^{2}\right) f^{(0)}+\left(f^{(0)}\right)^{\prime \prime}\right]+f_{e}^{4}\left(1-\lambda^{2}\right)\left(f^{(0)}\right)^{\prime \prime}=0,
\end{aligned}
$$

where, for brevity's sake, the following notations are adopted

$$
f_{e}^{2}=\left[(1-\lambda)^{2} f^{(0)}+\left(f^{(0)}\right)^{\prime \prime}\right]^{2}+4 \lambda^{2}\left(f^{(0)}\right)^{\prime 2}
$$




$$
C_{1}=4 \lambda[(\lambda-1) n+1], C_{2}=(\lambda-1) n[(\lambda-1) n+2] .
$$

The fourth order nonlinear ordinary differential equation (33) with the boundary conditions

$$
f^{(0)}(\theta= \pm \pi)=0, \quad\left(f^{(0)}\right)^{\prime}(\theta= \pm \pi)=0,
$$

defines a nonlinear eigenvalue problem in which the constant $\lambda$ is the eigenvalue and $f^{(0)}(\theta)$ is the corresponding eigenfunction. Note that one eigenvalue of the nonlinear eigenvalue problem (33), (34) is well-known. This eigenvalue $\lambda=n /(n+1)$ corresponds to the classical HRR-problem. However, this eigenvalue results in the contours of the TDZ given by the two-term and three-term asymptotic integrity parameter expansions which are substantially different from each other. Therefore it is necessary to find a new eigenvalue different from $\lambda=n /(n+1)$ and resulting in the shapes of the TDZ which are close to each other. The boundary value problem (the new eigenvalue problem for the eigenfunction $f^{(0)}(\theta)$ and eigenvalue $\lambda$ (or $\left.s=\lambda-1\right)$ ) formulated is solved numerically using the Runge-Kutta-Fehlberg and a shooting method. Numerical results are presented in Table 2.

Table 2

\begin{tabular}{|c|c|c|c|c|c|}
\hline \multirow{2}{*}{$n$} & \multirow{2}{*}{$m$} & \multicolumn{2}{|c|}{ Plane strain } & \multicolumn{2}{c|}{ Plane stress } \\
\cline { 3 - 6 } & & $s$ & $\left(f^{(0)}\right)^{\prime \prime}(0)$ & $s$ & $\left(f^{(0)}\right)^{\prime \prime}(0)$ \\
\hline 1 & 1 & -1.5 & -0.75 & -1.5 & -0.75 \\
\hline 2 & $0.7 n$ & -1.0 & -0.5 & -1.1540 & -0.5686 \\
\hline 3 & $0.7 n$ & -0.7716 & -0.4372 & -1.0 & -0.5 \\
\hline 4 & $0.7 n$ & -0.6684 & -0.4092 & -0.9133 & -0.4658 \\
\hline 5 & $0.7 n$ & -0.6179 & -0.3985 & -0.8580 & -0.4428 \\
\hline 6 & $0.7 n$ & -0.5901 & -0.3950 & -0.8197 & -0.4261 \\
\hline 7 & $0.7 n$ & -0.5732 & -0.3943 & -0.7919 & -0.4134 \\
\hline 8 & $0.7 n$ & -0.5621 & -0.3948 & -0.7708 & -0.4035 \\
\hline 9 & $0.7 n$ & -0.5543 & -0.3958 & -0.7543 & -0.3955 \\
\hline
\end{tabular}

The shapes of the TDZ obtained for the new stress asymptotics at large distances from the crack tip for plane strain and plane stress conditions are shown in Figures 7, 8 , where 1 - the contour given by the two-term asymptotic expansion of the integrity parameter, 2 - the contour given by the three-term asymptotic expansion of the integrity parameter, 3 - the contour given by the four-term asymptotic expansion of the integrity parameter.

Returning to the original variables one can estimate the size of the TDZ and find a law according to which the TDZ evolutes:

$$
r_{0}(t)=R(0) \tilde{C}^{-1 / s}(A t)^{-1 /(s m)} .
$$

Differentiating Eq. (35) with respect to time and replacing time by the radial coordinate through (35) give the rate of the TDZ:

$$
\frac{d r_{0}(t)}{d t}=-\frac{1}{s m}[R(0)]^{-s m} \tilde{C}^{m} A\left[r_{0}(t)\right]^{1+s m} .
$$



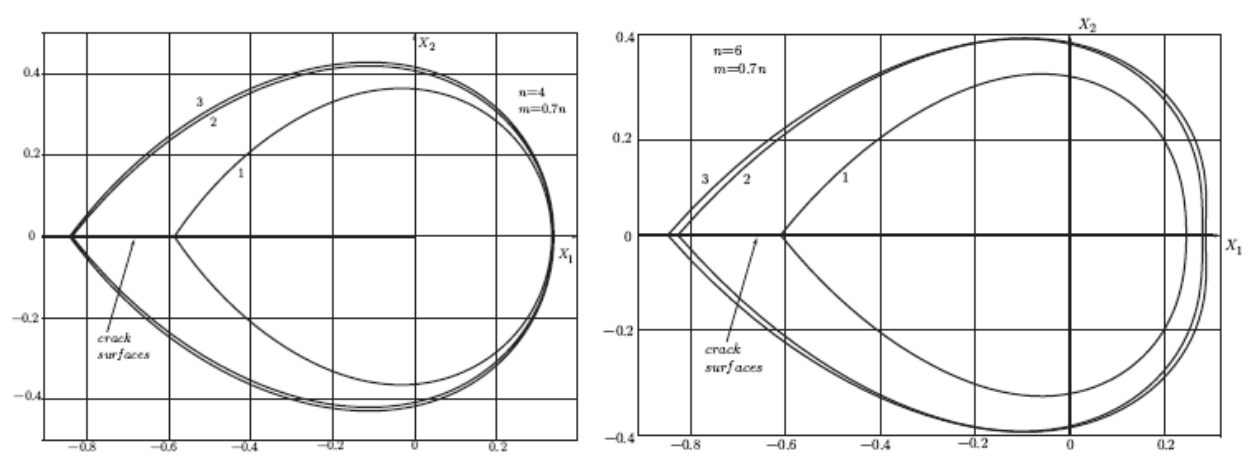

Fig. 6. The boundary of the TDZ for different values of material constants (plane strain conditions)
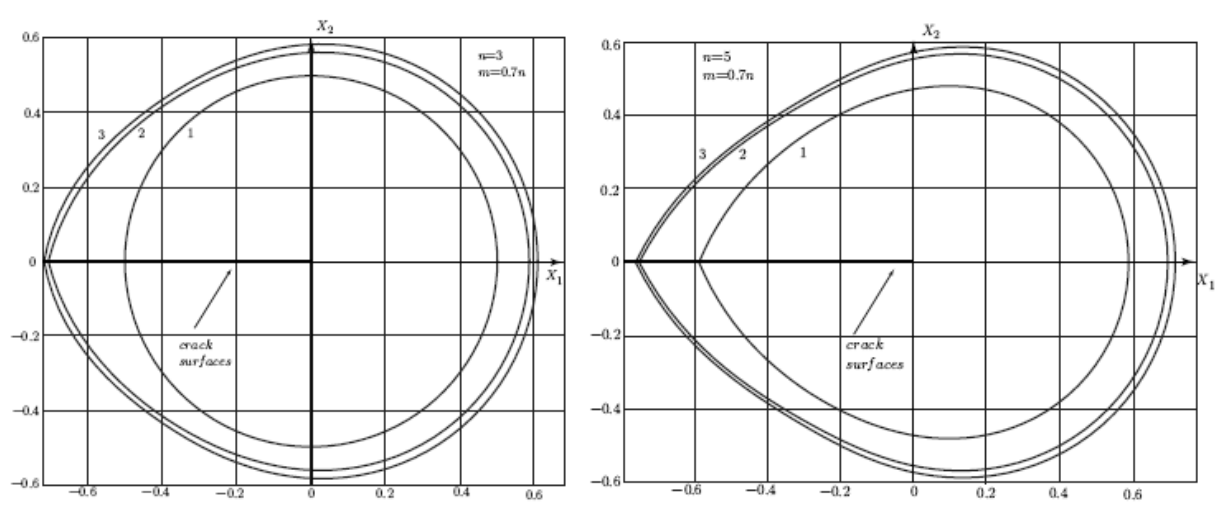

Fig. 7. The boundary of the TDZ for different values of material constants (plane stress conditions)

\section{CONCLUSIONS}

The effects of material damage on the asymptotic stress and creep strain rate fields of mode I and mode III cracks were analysed on the basis of continuum damage mechanics by postulating power-law creep damage theory.

Based on the similarity variable a stress analysis is carried out for the mode I crack under plane stress and plane strain conditions and for the mode III crack and assuming the existence of a totally damaged zone near the crack tip. It is found that the HutchinsonRice-Rosengren solution can't be used as the remote boundary condition and the actual far field stress is obtained. The shape of the totally damaged zone is given and analysed.

It is shown that the new far field stress asymptotics can be interpreted as the intermediate asymptotic valid for times and distances at which effects of initial and boundary conditions on the stress and damage distributions are lost.

Higher order fields for damaged nonlinear antiplane shear and tensile crack problems are analytically derived. The higher order fields obtained permit the shape of the totally damaged zone modelled in the vicinity of the crack tip to be determined more exactly. 


\section{REFERENCES}

[1] Z. H. Jin, R. C. Batra, Crack Shielding and Material Deterioration in Damaged Materials: an Antiplane Shear Fracture Problem, Archive of Applied Mechanics 68 (1998) 247-258.

[2] H. D. Bui, A. Ehrlacher, Propagation of Damage in Elastic and Plastic Solids. In: Francois D. (Ed.). Advances in Fracture Research, Pergamon Press, Oxford (1980) 533-551.

[3] H. D. Bui, Fracture mechanics: Inverse problems and solutions, Berlin: Springer (2006).

[4] V.I. Astafjev, T. V. Grigorova, Stress and Damage Distribution Near the Tip of a Crack Growing under Creep, Mechanics of Solids 30 (1995) 144-150.

[5] S. Murakami, T. Hirano and Y. Liu, Asymptotic Fields of Stress and Damage of a Mode I Creep Crack in Steady-State Growth, International Journal of Solids and Structures $\mathbf{3 7}$ (2000) 6203-6220.

[6] S. Murakami, Y. Liu and M. Mizuno, Computational methods for creep fracture analysis by damage mechanics, Computational Methods Applied Mechanics Engineering 183 (2000) 15-33.

[7] J. Zhao, X. Zhang, The Asymptotic Study of Fatigue Crack Growth Based on Damage Mechanics, Engineering Fracture Mechanics 50 (1995) 131-141.

[8] H. Riedel, Fracture at High Temperature, Springer, Berlin (1987).

[9] M. Anheuser, D. Gross, Higher order fields at crack and notch tips in power-law materials under longitudinal shear, Archive of Applied Mechanics 64 (1994) 509-518.

[10] L. V. Stepanova, Eigenvalues of the Antiplane-Shear Crack Problem for a Power-Law Material, Journal of Applied Mechanics and Technical Physics 49 (2008) 142-147.

[11] G. I. Barenblatt, Similarity, Self-similarity, and Intermediate Asymptotics, Consultants Bureau, New York (1979).

[12] G. I. Barenblatt, Scaling phenomena in fluid mechanics, Cambridge University Press (1994).

[13] P. L. Sachdev, Self-similarity and beyond. Exact solutions of nonlinear problems, New York: Chapman and Hall/CRC (2000).

[14] M. Ciavarella, M. Paggi and A. Carpinteri, One, no one and hundred thousand crack propagation laws: A generalized Barenblatt and Botvina dimensional analysis approach to fatigue crack growth, Journal of the Mechanics and Physics of Solids 56 (2008) 3416-3432.

Received August 7, 2009

\section{MộT LỜI GIẢI TIỆM CẬN TRUNG GIAN CHO BÀI TOÁN NỨT HƯ HỎNG TRONG CÁC BIẾN ĐỒNG DẠNG}

Lớp các lời giải tự đồng dạng cho bài toán nứt hư hỏng dão được quan tâm trong bài báo. Mô hình cơ bản được dựa trên cơ học của môi trường liên tục bị hư hỏng. Lý thuyết kinh điển hư hỏng dão của Kachanov-Rabotnov được sử dụng để nghiên cứu dáng điệu tiệm cận của hư hỏng trong vùng rất gần với vết nứt. Vùng bị hư hỏng hoàn toàn nơi mà các tham số thể hiện hư hỏng đạt tới giá trị tới hạn được giả sử tồn tại trong lân cận của vết nứt. Sử dụng các biến đồng dạng, ta thu được những lời giải tiệm cận cho dạng I và dạng III của bài toán nứt. ứng suất tiệm cận, tốc độ biến dạng dão và những vùng hư hỏng gần với vết nứt được phân tích bằng cách giải bài toán giá trị riêng phi tuyến dẫn tới một phân bố ứng suất mới. Hình dạng của vùng hư hỏng hoàn toàn chi phối bởi miền ứng suất mới sẽ được tìm và phân tích. 